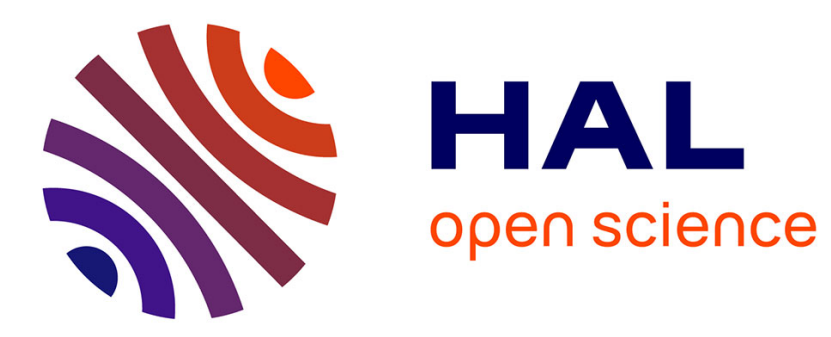

\title{
Structure Learning via Hadamard Product of Correlation and Partial Correlation Matrices
}

\author{
Karina Ashurbekova, Sophie Achard, Florence Forbes
}

\section{To cite this version:}

Karina Ashurbekova, Sophie Achard, Florence Forbes. Structure Learning via Hadamard Product of Correlation and Partial Correlation Matrices. EUSIPCO 2019 - 27th European Signal Processing Conference, Sep 2019, A Coruna, Spain. pp.1-5, 10.23919/EUSIPCO.2019.8902948 . hal-02290847

\section{HAL Id: hal-02290847 \\ https://hal.univ-grenoble-alpes.fr/hal-02290847}

Submitted on 19 Sep 2019

HAL is a multi-disciplinary open access archive for the deposit and dissemination of scientific research documents, whether they are published or not. The documents may come from teaching and research institutions in France or abroad, or from public or private research centers.
L'archive ouverte pluridisciplinaire $\mathbf{H A L}$, est destinée au dépôt et à la diffusion de documents scientifiques de niveau recherche, publiés ou non, émanant des établissements d'enseignement et de recherche français ou étrangers, des laboratoires publics ou privés. 


\section{Structure Learning via Hadamard Product of Correlation and Partial Correlation Matrices}

\author{
Karina Ashurbekova \\ GIPSA-lab, Inria \\ Univ. Grenoble Alpes \\ Grenoble, France \\ karina.ashurbekova@gipsa-lab.fr
}

\author{
Sophie Achard \\ CNRS, Grenoble INP, GIPSA-lab \\ Univ. Grenoble Alpes \\ Grenoble, France \\ sophie.achard@gipsa-lab.fr
}

\author{
Florence Forbes \\ Inria, CNRS, Grenoble INP, LJK \\ Univ. Grenoble Alpes \\ Grenoble, France \\ florence.forbes@inria.fr
}

\begin{abstract}
Structure learning is an active topic nowadays in different application areas, i.e. genetics, neuroscience. Classical conditional independences or marginal independences may not be sufficient to express complex relationships. This paper is introducing a new structure learning procedure where an edge in the graph corresponds to a non zero value of both correlation and partial correlation. Based on this new paradigm, we define an estimator and derive its theoretical properties. The asymptotic convergence of the proposed graph estimator and its rate are derived. Illustrations on a synthetic example and application to brain connectivity are displayed.
\end{abstract}

Index Terms-structure learning, conditional independence, marginal independence, Hadamard product, soft-thresholding, brain connectivity

\section{INTRODUCTION}

Finding relationships among a large number of random variables is a difficult problem that has generated a substantial interest in recent years. This is due to the large number of application areas where relationships are important features to extract. For example, in neuroscience, brain networks providing relationships between brain regions, have brought new understandings on the brain mechanisms under a pathological condition. Other examples can be found in genetics, medicine, finance, social networks. Graphical representations of probabilistic relationships have been extensively studied in machine learning and statistics. In particular, the so-called Graphical Models [11] are a way to represent probability density functions (pdf) that makes explicit factorization properties of the pdf and as a consequence helps in designing efficient inference algorithms. Given a set of $p$ variables $Y=\left(Y_{1}, Y_{2}, \cdots Y_{p}\right)$, one important issue in practice, is therefore how to infer such a graph structure from a $n$-sample of $\left(Y_{1}, Y_{2}, \cdots Y_{p}\right)$. The appropriate procedure may of course depend on additional assumptions on the joint pdf of the $Y_{i}$ 's and on the type of relationships that is seek. Among the various dependence measures, conditional dependence is the most frequent as it is often seen as a more suitable concept to catch direct link than standard dependence, referred to as marginal dependence. Conditional independence of two variables $Y_{i}$ and $Y_{j}$ is defined as the independence of these two variables after regressing out the other variables. It is denoted by $Y_{i} \perp Y_{j} \mid Y_{-(i, j)}$ where $Y_{-(i, j)}$ is the set of variables $Y$ after removing $Y_{i}$ and $Y_{j}$.
A graph $G=(V, E)$ can then be constructed. The set of nodes $V$ corresponds to the $p$ variables in $Y$, and the edge set $E \subset V \times V$ is defined as follows

$$
Y_{i} \perp Y_{j} \mid Y_{-(i, j)} \Leftrightarrow(i, j) \notin E .
$$

We will refer to this graph as a partial correlation graph.

Similarly, marginal independence of two variables $Y_{i}$ and $Y_{j}$ is the standard probabilistic independence denoted by $Y_{i} \perp Y_{j}$. A graph can also be defined as,

$$
Y_{i} \perp Y_{j} \Leftrightarrow(i, j) \notin E .
$$

We will refer to this graph as a correlation graph. The two dependence measures and therefore the two graphs are not equivalent nor one is included in the other (eg. [8]). This is illustrated in Figure 1. Figure 1 (a) illustrates the so-called common effect phenomenon or $\mathrm{v}$-structure. Using the Bayesian network representation, this graph corresponds to the joint distribution $p\left(x_{1}, x_{2}, x_{3}\right)=p\left(x_{3} \mid x_{2}, x_{1}\right) \quad p\left(x_{1}\right) \quad p\left(x_{2}\right)$ for which it is easy to check that $X_{2}$ and $X_{1}$ are independent but not conditionally independent. The correlation graph is the one to use in Figure 1 (a) to find the v-structure, while a partial correlation graph would give a triangle for this pdf. Conversely for the pdf represented in Figure 1 (b), $p\left(x_{1}, x_{3}, x_{4}\right)=p\left(x_{3} \mid x_{1}\right) p\left(x_{4} \mid x_{1}\right) p\left(x_{1}\right), X_{3}$ and $X_{4}$ are marginally dependent but not conditionally independent. The partial correlation graph is the one to use while the correlation graph would be a triangle. More generally, for more complex graphs that would mix the two phenomenons, neither covariance graphs nor precision graphs would express our understanding of the variables relationships in a satisfying manner (see Figure 1 (c)). However, the intersection of the two graphs seems promising in structure learning.

In this work, we therefore propose to consider simultaneously both conditional and marginal dependencies and to address the problem of estimating them from observed data.

If the joint pdf of $\left(Y_{1}, Y_{2}, \cdots Y_{p}\right)$ is Gaussian, i.e. $Y \sim$ $N_{p}(\mu, \Sigma)$, then conditional independence can be read directly from the precision matrix $\Theta=\Sigma^{-1}$ of the pdf. This provides a relatively straightforward way to assess conditional independencies from a data sample, by estimating the empirical precision matrix. Similarly, marginal independence can be read on the covariance matrix $\Sigma$ which can also be estimated 
(a) common effect

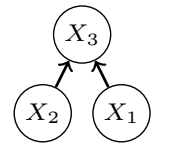

(b) common cause

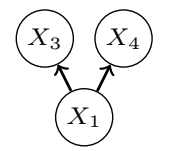

(c) mixing model

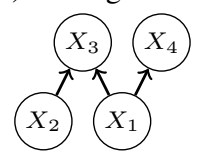

Fig. 1: Illustration of the common cause, common effect phenomena and the combination of common cause and common effect phenomena where neither correlation nor partial correlation can retrieve the real structure of the graph. This problem is a standard issue encountered in directed graphs exploration.

empirically. Most popular approaches resort to penalized likelihood estimation with the idea of favoring sparse matrices. Reviews of recent results including methods adapted for highdimensional setting and heavy-tailed distributions can be found in [6], [8]. Starting with graphical lasso (glasso) [10], penalized likelihood approaches based on $L_{1}$-norm of the precision matrix $\Theta$ have been increasingly popular [1], [23]. Several estimators have been studied based on the use of Dantzig selector [22], CLIME algorithm [5] or thresholding operator [19]. In contrast to most other proposals, the estimator proposed in [19] has a closed-form solution as long as it is invertible. Similarly, [3] uses a glasso approach for the covariance matrix while recent progress on this problem has been made in [18] with a new optimization method based on coordinate descent. Other approaches [2], [14] consider generalized thresholding of the covariance matrix to get a sparse estimate. These methods have good theoretical properties and are computationally efficient. Finally, recent developments based on graph signal processing have brought new perspectives on this problem [7], [13]. Solutions are based on the study of eigenvalues and eigenvectors of Laplacian matrix using either correlation or partial correlation as inputs.

However, to our knowledge the existing methods use either $\Theta$ (conditional dependencies) or $\Sigma$ (marginal dependencies) to infer the graph. The purpose of the present work is to develop a method to construct a graph where the absence of an edge between $Y_{i}$ and $Y_{j}$ corresponds to the absence of one of the properties: partial correlation or correlation. The graph is then defined by:

$$
Y_{i} \perp Y_{j} \mid Y_{-(i, j)} \text { or } Y_{i} \perp Y_{j} \Leftrightarrow(i, j) \notin E \text {. }
$$

This paper is organized as follows. We briefly give an overview of the related work. A new definition of the structure of the graph using Hadamard product is introduced in section III. Theoretical properties of the proposed estimator are derived in section IV and an illustration is presented in section V. Finally, a validation on real data is described in section VI.

\section{PRIOR WORK}

Despite the very large number of existing papers dealing with conditional independence (i.e. classical Gaussian graphical models), there are few papers studying the common cause - common effect problem.
Using specific conditions on sparsity, [9] showed that positions of zeros in partial correlation and correlation matrices are equal. As a consequence for these conditions, both dependence measures, partial correlation or correlation, are estimating the same graphs. However, hypotheses on sparsity are hard to check, and we argue that they are too restrictive in many applications.

In the framework of Bayesian networks, heuristic approaches have been developed to iteratively decompose the graph and to find the v-structures. In [21] the authors propose to infer the dependence structure after identifying subset of nodes with a possible v-structure. This approach may be very costly as it is not providing an analytic solution. Alternative approaches based on graph signal processing tend to propose an optimization framework independent of the input matrices correlation or partial correlation. In [16], the authors derive a criterion based on eigenvectors decomposition of covariance matrix. Its minimization under the constraint of sparsity is achieved corresponding to either correlation or partial correlation. Using this method it is possible to retrieve the two examples given in Fig. 1 (a) or Fig. 1 (b). However for Fig. 1 (c), this method will not give the correct solution because of the combination of the two phenomena.

\section{HADAMARD STRUCTURE LEARNING}

In this section, we describe a novel method for defining graphical models in the way that the absence of edge between two nodes means that either the correlation of these variables equal to zero or the partial correlation of these variables given the rest equal to zero. The idea is to incorporate both correlation matrix $R=\left(r_{i j}\right)$ and partial correlation matrix $\Gamma=\left(\gamma_{i j}\right)$ in one product. We focus on Hadamard product for its tractability and ability to reveal the absence of at least one of the properties among partial correlation or marginal correlation: $\mathcal{H}=R \circ \Gamma, \mathcal{H}=\left(h_{i j}\right)$. Therefore, $h_{i j}$ have zero on the place $i j$ if at least one of $r_{i j}$ and $\gamma_{i j}$ equals to zero. The graph (3) is then defined by zeros of matrix $\mathcal{H}$ and we call this graph a Hadamard graph. The edges of this graph correspond to non-zeros values for both partial and marginal correlations.

As an example of the application of this new definition on real data, we used brain data using functional Magnetic Resonance Imaging (fMRI). The details of the preprocessing of the data is explained in [17]. Figure 2 is displaying the obtained correlation graph (a), partial correlation graph (b) and Hadamard graph (c). Each graph is obtained by defining 40 edges corresponding to the first 40th highest absolute values of respectively $R$ (Figure 2 (a)), $\Gamma$ (Figure 2 (b)) and $R \circ \Gamma$ (Figure 2 (c)). Different positioning of the edges on the structure of the brain are obtained. Correlation graph is highlighting more short-range connections (clustering of regions close in space), whereas partial correlation is capturing long-range connections (connection of regions that are far from each other). These two features can be related to the cost-efficiency trade-off of the brain [4]. It is assumed that the brain networks are constructed in order to both optimize the efficiency (facility to connect 
(a)

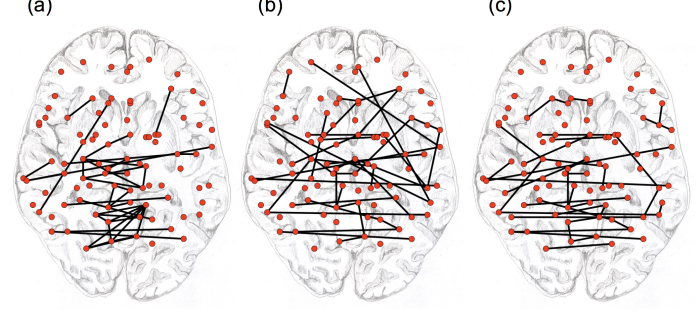

Fig. 2: Structure learning on brain graphs using fMRI data: (a) correlation graph; (b) partial correlation graph; (c) Hadamard graph. Short-range connections are prevalent in (a) whereas edges in (b) are mostly long-range connections. (c) is a combination of (a) and (b).

any two regions of the brain), and the cost (the cost to build structural connections). The Hadamard graph can be seen as an illustration of this trade-off where both short-range and long-range connections are present in the brain.

\section{ESTIMATION OF HADAMARD GRAPHS}

For now, for simplicity, we assume that the sample correlation matrix $\widehat{R}$ is invertible. To recover the Hadamard product parameter $\mathcal{H}$ we propose the following estimator which allows us to regularize the number of connections defined as the nonzero elements in $\mathcal{H}$ :

$$
\begin{aligned}
\arg \min _{\mathcal{H}} & \|\mathcal{H}\|_{1, \text { off }} \\
\text { s.t.: } & |\mathcal{H}-\widehat{\mathcal{H}}|_{\infty, \text { off }} \leqslant \lambda,
\end{aligned}
$$

where $\widehat{\mathcal{H}}=\widehat{R} \circ \widehat{\Gamma}$ and $\widehat{\Gamma}$ is the estimator of the partial correlation matrix obtained by normalizing $\widehat{R}^{-1}$, for matrix $A:|A|_{\infty . \text { off }}=\max _{i \neq j}\left|A_{i j}\right|,\|A\|_{1, \text { off }}=\sum_{i \neq j}\left|A_{i j}\right|$. Note that similar approaches were considered in [19] and [20] but for the estimation of the precision and covariance matrices respectively. Optimization problem (4) is decomposable into independent element-wise sub-problems, where each subproblem corresponds to soft-thresholding of $\mathcal{H}$ [20]. Thus, the estimator (4) is available in closed-form: $\widehat{\mathcal{H}}_{\lambda}=T_{\lambda}(\widehat{\mathcal{H}})$, where $\left[T_{\lambda}(u)\right]_{i j}=\operatorname{sign}\left(u_{i j}\right) \max \left(\left|u_{i j}\right|-\lambda, 0\right)$. Now we derive theoretical properties for the thresholding of the Hadamard product.

We consider the following uniformity class of "approximately sparse" correlation matrices invariant under permutations presented in papers [2] and [14]:

$$
\mathcal{U}_{\tau}\left(q_{0}, c_{o}(p)\right)=\left\{R, \sum_{j=1}^{p}\left|r_{i j}\right|^{q_{0}} \leq c_{0}(p) \forall i\right\}
$$

for $0 \leq q_{0}<1$. When $q_{0}=0, \sum_{j=1}^{p}\left|r_{i j}\right|^{q_{0}}=\sum_{j=1}^{p} \mathbb{1}\left(r_{i j} \neq\right.$ $0)$ which corresponds to the class of sparse matrices ( $\mathbb{1}$ means the indicator function). The dependence of $c_{0}(p)$ on the dimension $p$ allows to be less restricted on the sparsity [14]. We assume that the partial correlation matrix also belongs to the class of "approximately sparse" matrices invariant under permutations:

$$
\mathcal{U}_{\tau}\left(q_{1}, c_{1}(p)\right)=\left\{\Gamma, \sum_{j=1}^{p}\left|\gamma_{i j}\right|^{q_{1}} \leq c_{1}(p) \forall i\right\}
$$

for $0 \leq q_{1}<1$. Then the Hadamard product of the correlation and partial correlation matrices is also in the class of "approximately sparse" matrices:

Proposition 1. The Hadamard product $\mathcal{H}$ of correlation matrix $R$ in the class $\mathcal{U}_{\tau}\left(q_{0}, c_{o}(p)\right)$ and partial correlation matrix $\Gamma$ in the class $\mathcal{U}_{\tau}\left(q_{1}, c_{1}(p)\right)$ also belongs to the class of "approximately sparse" matrices $\mathcal{U}_{\tau}\left(q_{2}, c_{2}(p)\right)$

for $0 \leq q_{2}<1$.

Proof. Let us assume $q_{2}=\frac{1}{2} \min \left(q_{0}, q_{1}\right)$ and $c_{2}(p)=$ $\max \left(c_{0}(p), c_{1}(p)\right)$. Then for $i, 1 \leq i \leq p$,

$$
\begin{aligned}
& \sum_{j=1}^{p}\left|h_{i j}\right|^{q_{2}}=\sum_{j=1}^{p}\left|r_{i j} \gamma_{i j}\right|^{q_{2}}=\sum_{j=1}^{p}\left|r_{i j}\right|^{q_{2}}\left|\gamma_{i j}\right|^{q_{2}} \\
& \leq \sqrt{\sum_{j=1}^{p}\left|r_{i j}\right|^{2 q_{2}}} \sqrt{\sum_{j=1}^{p}\left|\gamma_{i j}\right|^{2 q_{2}}} \leq \sqrt{\sum_{j=1}^{p}\left|r_{i j}\right|^{q_{0}}} \sqrt{\sum_{j=1}^{p}\left|\gamma_{i j}\right|^{q_{1}}} \\
& \leq\left(c_{0}(p)\right)^{1 / 2}\left(c_{1}(p)\right)^{1 / 2} \leq \max \left(c_{o}(p), c_{1}(p)\right)
\end{aligned}
$$

This means that the Hadamard product belongs to

$$
\mathcal{U}_{\tau}\left(q_{2}, c_{2}(p)\right)=\left\{H, \sum_{j=1}^{p}\left|h_{i j}\right|^{q_{2}} \leq c_{2}(p) \forall i\right\}
$$

for some $0 \leq q_{2}<1$.

The following theorem provides the convergence rate for $T_{\lambda}(\widehat{\mathcal{H}})$ in the matrix operator norm, for matrix $A$ : $\|A\|^{2}=$ $\lambda_{\max }\left(A A^{T}\right)$, where $\lambda_{\max }\left(A A^{T}\right)$ represents the largest eigenvalue of matrix $A A^{T}$.

Theorem 1. Suppose the distribution of the data is Gaussian with Hadamard product $\mathcal{H}=R \circ \Gamma$. Then, uniformly on $\mathcal{U}_{\tau}\left(q_{2}, c_{2}(p)\right)$, for sufficiently large $M^{\prime}$, if $\lambda=M^{\prime} \sqrt{\frac{\log p}{n}}$ and $\frac{\log p}{n}=o(1)$, then:

$$
P\left[\left\|T_{\lambda}(\widehat{\mathcal{H}})-\mathcal{H}\right\|<c_{2}(p)\left(\frac{\log p}{n}\right)^{\left(1-q_{2}\right) / 2}\right] \geq 1-\epsilon_{n, p},
$$

where $\epsilon_{n, p}$ is a deterministic sequence that decreases to zero as $n, p \rightarrow \infty$.

Proof. By examining the proof of Theorem 1 in [14], the only property we need to prove is the following:

$$
\begin{gathered}
P\left[\max _{i j}\left|h_{i j}-\widehat{h}_{i j}\right|<\frac{\log p}{n}\right] \geq 1-\epsilon_{n, p} \\
\max _{i j}\left|h_{i j}-\widehat{h}_{i j}\right|=\max _{i j}\left|r_{i j} \gamma_{i j}-\widehat{r}_{i j} \widehat{\gamma}_{i j}\right| \leq \\
\leq \max _{i j}\left|-\widehat{r}_{i j} \widehat{\gamma}_{i j}+\widehat{r}_{i j} \gamma_{i j}\right|+\max _{i j}\left|-\widehat{r}_{i j} \gamma_{i j}+r_{i j} \gamma_{i j}\right|= \\
=\max _{i j}\left|\widehat{r}_{i j}\left(\gamma_{i j}-\widehat{\gamma}_{i j}\right)\right|+\max _{i j}\left|\gamma_{i j}\left(r_{i j}-\widehat{r}_{i j}\right)\right| \leq \\
\leq \max _{i j}\left|\widehat{r}_{i j}\right| \max _{i j}\left|\gamma_{i j}-\widehat{\gamma}_{i j}\right|+\max _{i j}\left|\gamma_{i j}\right| \max _{i j}\left|r_{i j}-\widehat{r}_{i j}\right|
\end{gathered}
$$


Applying theorem 1 [14] to each of the two terms of the righthand side of the above inequality finishes the proof.

Remark 1. Sample covariance (correlation) matrices are not invertible unless the sample size $n$ is larger than the number of variables $p$ and we cannot compute a partial correlation matrix using the sample correlation matrix $R$. This issue can be solved using the thresholding operator of sample correlation matrix, i.e. $\Gamma$ can be derived from $\left[T_{\nu}(\widehat{R})\right]^{-1}$. This idea was implemented in [20] and under additional assumptions on precision matrix it is shown that this estimator is well-defined and well-behaved.

\section{ILLUSTRATION ON SIMULATED DATA}

We illustrate the behaviour of soft-thresholding operator of Hadamard product of estimated correlation matrix and partial correlation matrix in terms of the recovering of the sparsity pattern. In order to confirm the usefulness of our framework, we construct an example where neither correlation graph nor partial correlation graph could reconstruct the correct structure. First, we simulate two positive definite matrices $R_{1}$ and $R_{2}$ of size 100 each using models 1 and 2 respectively. Model 1: "Triangular" correlation, $r_{i j}=(1-((|i-j|) / k))_{+}$, for $k=[p / 4]$. Model 2: inverse of "Triangular" correlation.

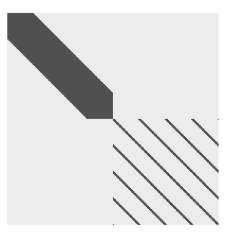

(a)

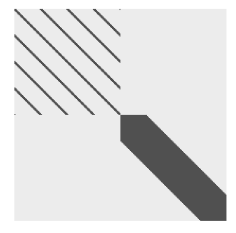

(b)

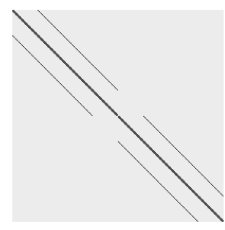

(c)
Fig. 3: Adjacency matrix of (a) correlation, (b) partial correlation and (c) Hadamard matrix.

Then we take the true correlation $R$ matrix of size 200 as a block-diagonal matrix of $R_{1}$ and $R_{2}$. Model 1 is a standard test case in the literature. The adjacency matrices of the true correlation matrix $R$, partial correlation matrix and their Hadamard product are presented in Figure 3. We generated $n=300$ independent and identically distributed $p$ variate normal random vectors with mean 0 and correlation matrix equal to $R$. The ability to recover sparsity patterns of the true Hadamard product was evaluated via ROC curves.

We compare two methods: soft-thresholding of Hadamard product $\widehat{\mathcal{H}}$, i.e. $T_{\lambda}(\widehat{\mathcal{H}})=T_{\lambda}(\widehat{R} \circ \widehat{\Gamma})$, where $\widehat{R}$ is the sample correlation matrix and $\widehat{\Gamma}$ is the estimator of the partial correlation matrix obtained by normalizing the matrix $\widehat{R}$; Hadamard product of soft-thresholding of correlation matrix $\widehat{R}$ and softthresholding of partial correlation matrix $\widehat{\Gamma}$, i.e. $T_{\lambda}(\widehat{\mathcal{R}}) \circ T_{\lambda}(\widehat{\Gamma})$. The whole process was run 50 times. The corresponding ROC in Figure 4 shows that result for $T_{\lambda}(\widehat{\mathcal{H}})$ outperforms the result for $T_{\lambda}(\widehat{\mathcal{R}}) \circ T_{\lambda}(\widehat{\Gamma})$ for the FPR greater then $6 \%$.

\section{VALIDATION ON REAL DATA}

In order to validate our approach on real data, we tested our graph structure learning approach on brain signals using

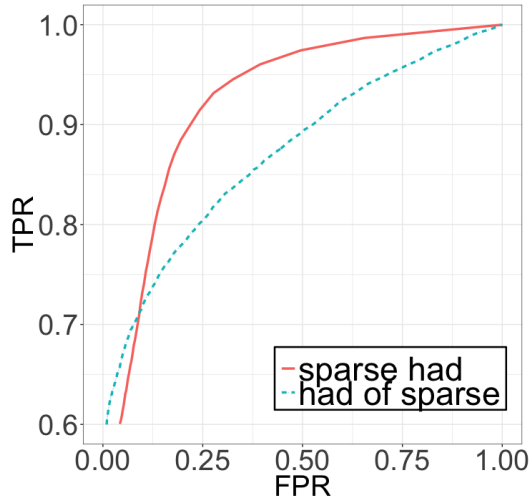

Fig. 4: ROC curve representing the performance of $T_{\lambda}(\widehat{\mathcal{R}}) \circ$ $T_{\lambda}(\widehat{\Gamma})$ and $T_{\lambda}(\widehat{\mathcal{H}})$. The ROC curve is created by plotting the true positive rate (TPR) against the false positive rate (FPR) at various threshold settings $\lambda$. For any estimated matrix $M_{\lambda}=$ $\left(m(\lambda)_{i j}\right)$ and the true Hadamard matrix $H=\left(h_{i j}\right)$ TPR and FPR are defined as: TPR $=\frac{\#\left\{(i, j): m(\lambda)_{i j} \neq 0 \text { and } h_{i j} \neq 0\right\}}{\#\left\{(i, j): h_{i j} \neq 0\right\}}$ and $\mathrm{FPR}=\frac{\#\left\{(i, j): m(\lambda)_{i j} \neq 0 \text { and } h_{i j}=0\right\}}{\#\left\{(i, j): h_{i j}=0\right\}}$.

fMRI. This provides brain connectivity networks that are used in the identification of cognitive impairments for example. As ground truth for evaluating brain connectivity networks is not available, we used test-retest datasets (see [17] for details) to quantify the performances of the different approaches. 100 subjects were scanned twice in two different sessions. The reliability of each structure learning approach is evaluated by measuring the difference of graph properties for the two sessions obtained for the same subject. For each datasets, we compute a graph using four different approaches: $\widehat{R}, \widehat{\Gamma}$, $\widehat{\mathcal{H}}=\widehat{R} \circ \widehat{\Gamma}$ and partial correlation matrix obtained from glasso algorithm; the tuning parameter $\lambda$ in glasso algorithm for each subject in each session was obtained by crossvalidation with $k=5$ folds. In order to produce a fixed number of edges, we apply the soft-thresholding for each of the matrix. Thus we obtain an adjacency matrix that defines an unweighted graph for each subject in each session. We compute a graph metric called "global efficiency" which is related to the communication efficiency of a node $i$ with all other nodes (detailed information can be found in [15]). For each graph we obtain one value of this parameter. Accordingly, there are 200 values of global efficiency (one per each subject in each session) for fixed value of percentage of edges in the graphs. To evaluate the reliability of each method we compute intraclass correlation coefficient $(I C C)$ which is based on the comparison of the within-subject and between-subject variability. The Figure 5 represents the scheme of computing the $I C C$ for fixed number of edges. $I C C$ is close to 0 when the reliability is low, and close to 1 when the reliability is high. Note that $I C C$ may take negative values when the variance within subjects is larger than between subjects. This is due to statistical errors given a particular dataset and should be considered as a non reliable estimation.

The results are shown in Figure 6. It is clear that methods 


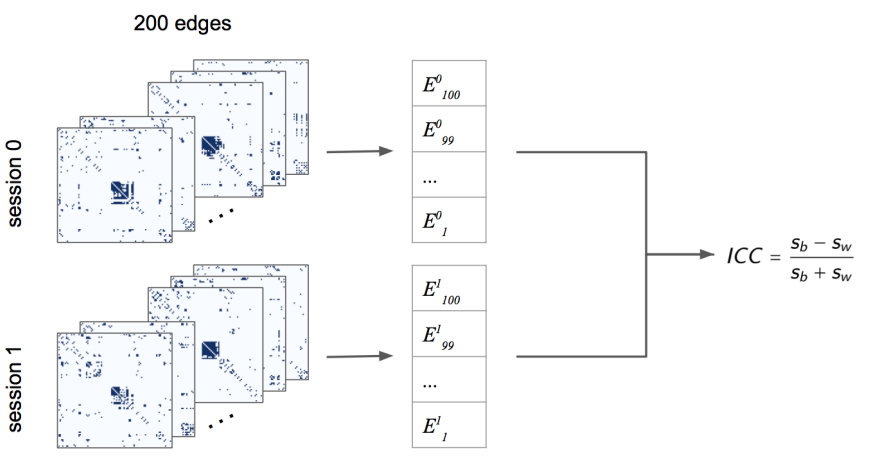

Fig. 5: The computation of $I C C$ for global efficiency for fixed number of edges equal to $200 . E_{1}^{0}, \ldots E_{100}^{0}, E_{1}^{1}, \ldots, E_{100}^{1}$ are values of global efficiency for each subject in both sessions; $s_{b}$ is the variance between subjects, $s_{w}$ is the variance within subjects in 2 sessions; $I C C=\left(s_{b}-s_{w}\right) /\left(s_{b}+s_{w}\right)$ (see [17] for more details about computing $s_{b}$ and $s_{w}$ ).

based on partial correlation are the least reliable. Using glasso improves the reliability however correlation approaches are still better. For this datasets, Hadamard approach gives similar results as sample correlation, which means that brain connectivity networks seem to be better characterized by marginal dependence. The highest value of ICC is obtained when using Hadamard graphs for $30 \%$ of edges present in the graphs. When there are no hypotheses on the dependence structure of the graphs, these results show that Hadamard graph is a good trade off for structure learning.

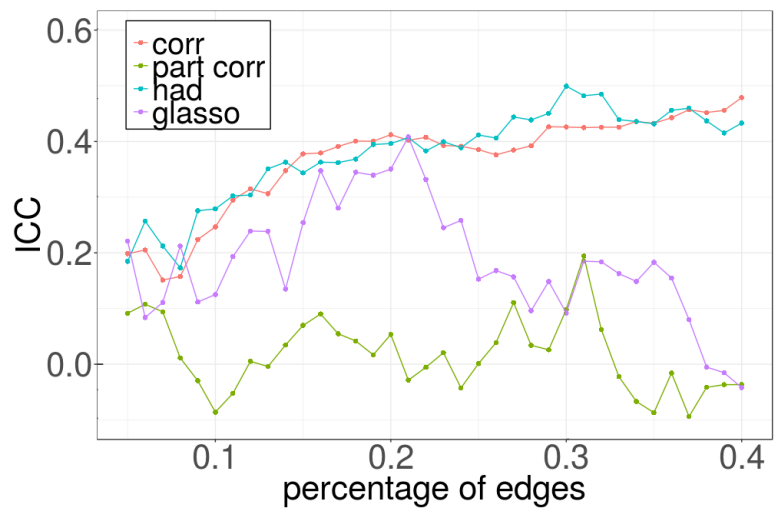

Fig. 6: Reliability measures using $I C C$ for global efficiency for the sample correlation matrix $\widehat{R}$, the partial correlation matrix $\widehat{\Gamma}=\widehat{R}^{-1}$, Hadamard product $\widehat{\mathcal{H}}$ and partial correlation matrix obtained from glasso algorithm

\section{CONCLUSION}

We introduce a new graph construction method taking into account both conditional and marginal independences. We derive a convergence bound for the proposed estimator and show on synthetic data the ability of the estimator to recover the sparsity patterns of the true matrix. Our approach was applied on brain signals and showed better results than classical correlations or partial correlations. A nice advantage of our method is its simplicity where we only need to choose the estimation procedure for correlation and partial correlation matrices. In perspective, the estimation procedure for these matrices can be chosen to improve robustness to non-Gaussian data, for example with Kendall tau. Also, it is possible to use estimation based on shrinkaging of covariance matrix for highdimensional case [12].

\section{REFERENCES}

[1] O. Banerjee, L. El Ghaoui, and A. d'Aspremont. Model selection through sparse maximum likelihood estimation for multivariate Gaussian or binary data. J. Mach. Learn. Res., 9(Mar):485-516, 2008.

[2] P. J Bickel, E. Levina, et al. Covariance regularization by thresholding. The Annals of Statistics, 36(6):2577-2604, 2008.

[3] J. Bien and R. Tibshirani. Sparse estimation of a covariance matrix. Biometrika, 98(4):807-820, 2011.

[4] E. Bullmore and O. Sporns. The economy of brain network organization. Nature Reviews Neuroscience, 13(5):336, 2012.

[5] T. Cai, W. Liu, and Xi Luo. A constrained 1 minimization approach to sparse precision matrix estimation. Journal of the American Statistical Association, 106(494):594-607, 2011.

[6] T. Cai, Z. Ren, and H. Zhou. Estimating structured high-dimensional covariance and precision matrices: Optimal rates and adaptive estimation. Electron. J. Stat., 10(1):1-59, 2016.

[7] X. Dong, D. Thanou, P. Frossard, and P. Vandergheynst. Learning laplacian matrix in smooth graph signal representations. IEEE Transactions on Signal Processing, 64(23):6160-6173, 2016.

[8] J. Fan, Y. Liao, and H. Liu. An overview of the estimation of large covariance and precision matrices. Econom. J., 19(1), 2016.

[9] S. Fattahi and S. Sojoudi. Graphical lasso and thresholding: Equivalence and closed-form solutions. arXiv preprint arXiv:1708.09479, 2017.

[10] J. Friedman, T. Hastie, and R. Tibshirani. Sparse inverse covariance estimation with the graphical lasso. Biostatistics, 9(3):432-441, 2008.

[11] N. Friedman and D. Koller. Probabilistic graphical models: Principles and techniques, 2009.

[12] O. Ledoit and M. Wolf. A well-conditioned estimator for largedimensional covariance matrices. Journal of multivariate analysis, 88(2):365-411, 2004.

[13] J. Mei and J. Moura. Signal processing on graphs: Estimating the structure of a graph. In International Conference on Acoustics, Speech and Signal Processing, pages 5495-5499. IEEE, 2015.

[14] A. J. Rothman, E. Levina, and Ji Zhu. Generalized thresholding of large covariance matrices. Journal of the American Statistical Association 104(485):177-186, 2009.

[15] M. Rubinov and O. Sporns. Complex network measures of brain connectivity: uses and interpretations. Neuroimage, 52(3):1059-1069, 2010 .

[16] S. Segarra, A. G. Marques, G. Mateos, and A. Ribeiro. Network topology inference from spectral templates. IEEE Transactions on Signal and Information Processing over Networks, 3(3):467-483, 2017.

[17] M. Termenon, C. Delon-Martin, A. Jaillard, and S. Achard. Reliability of graph analysis of resting state fMRI using test-retest dataset from the human connectome project. Neuroimage, 142(15):172-187, 2016.

[18] H. Wang. Coordinate descent algorithm for covariance graphical lasso. Statistics and Computing, 24(4):521-529, 2014

[19] E. Yang, A. C. Lozano, and P. Ravikumar. Elementary estimators for graphical models. In Advances in neural information processing systems, pages 2159-2167, 2014.

[20] E. Yang, A. C. Lozano, and P. Ravikumar. Elementary estimators for sparse covariance matrices and other structured moments. In International conference on machine learning, pages 397-405, 2014.

[21] R. Yehezkel and B. Lerner. Bayesian network structure learning by recursive autonomy identification. Journal of Machine Learning Research, 10(Jul):1527-1570, 2009.

[22] M. Yuan. High dimensional inverse covariance matrix estimation via linear programming. Journal of Machine Learning Research, 11(Aug):2261-2286, 2010.

[23] M. Yuan and Y. Lin. Model selection and estimation in the Gaussian graphical model. Biometrika, 94(1):19-35, 2007. 